Artigo original

\title{
Implementação do programa nacional de melhoria do acesso e da qualidade da atenção básica (PMAQ-AB)
}

Implementation of the National Program for Access and Quality Improvement in Primary Care (PMAQ-AB)

Rosimeri Telles $^{1}$, Leni Dias Weigelt ${ }^{1}$, Maristela Soares Rezende ${ }^{1}$, Suzane Beatriz Frantz $\mathrm{Krug}^{1}$, Ronise Ferreira ${ }^{1}$, Amanda Luisa Kessler ${ }^{1}$, Lilian Pereira de Barros ${ }^{1}$, Ana Carolina Jühlich $^{1}$, Gabriela Mendes da Silva Flores ${ }^{1}$

${ }^{1}$ Universidade de Santa Cruz do Sul, Santa Cruz do Sul, RS, Brasil.

Submissão: 08/08/2016

Aceite: 20/09/16

rositelles13@gmail.com

\section{RESUMO}

Justificativa e Objetivo: investigar sobre a metodologia de aplicação do Programa Nacional de Melhoria do Acesso e da Qualidade da Atenção Básica (PMAQ-AB): sua avaliação pelos profissionais de saúde, seus resultados e seu impacto na organização e na assistência a saúde, sob, a ótica dos trabalhadores da saúde. Métodos: Pesquisa qualitativa, de caráter exploratório descritivo, com 21 trabalhadores de unidades de Estratégia de Saúde da Família (ESF) de um município da 28 Região de Saúde - RS com 17 ESFs cadastradas no Departamento de Atenção Básica-MS. Destas, oito possuem certificação como Equipe de Saúde Bucal. A coleta de dados ocorreu em documentos na Secretaria Municipal de Saúde e por meio de entrevistas. Constituíram a amostra o gestor de saúde e um representante por categoria de cada ESF que participou de alguma fase do PMAQ. A análise de conteúdo orientou a organização das informações e dos resultados em temas: PMAQ na visão dos trabalhadores, a metodologia de aplicação, os resultados e mudanças nas unidades de saúde com a implantação do programa. Resultados: Foram entrevistados dois médicos, cinco enfermeiros, sete técnicos de enfermagem, seis odontólogos e uma nutricionista. Identificouse a diversidade de percepções dos trabalhadores quanto à implementação do PMAQ-AB e o descontentamento por falta de retorno da gestão em relação aos resultados da avaliação. Conclusões: A falta de conhecimento dos profissionais sobre a metodologia de aplicação deste programa ficou evidente, pois grande parte dos entrevistados não consegue informar sobre as fases de implementação e o desenvolvimento das mesmas na sua ESF.

DESCRITORES: Avaliação em Saúde. Educação em Saúde. Assistência à saúde. Saúde Pública.

\section{ABSTRACT}

Justification and Objective: investigate about the methodology of implementing the National Program for Access and Quality Improvement in Primary Care (PMAQ-AB): review 
by health professionals, their results and their impact on the organization and assistance to health from the perspective of health workers. Method: Qualitative research, with exploratory descriptive study, were 21 workers the health of strategies units of family of a municipality $28^{\text {th }}$ Health Region - Rio Grande do Sul (RS), with 17 Family Health Strategy facilities registered in the Primary Care Department-Health Ministry. Of these 17 Family Health Strategy facilities, 8 have Team Dental Health certification. The data collection was from municipal health documents through interviews. Constituted the sample, the health manager and a representative of each category by the ESF to participate in any phase of PMAQ. The content analysis guided the organization of the information and the results in topics: PMAQ in the view of workers, the application methodology, the results and changes in the health facilities with program implementation. Results: Two doctors, five nurses, seven nursing technicians, six dentists and a nutritionist were interviewed. It identified the diversity of perceptions of health workers on the implementation of PMAQ - $\mathrm{AB}$, in the health facilities where they work and dissatisfaction for lack of feedback of the management in relation to the evaluation results. Conclusions: The lack of knowledge of professionals on the implementation methodology of this program was evident, since most of the respondents can not report on the implementation stages and their development in their Family Health Strategy.

KEYWORDS: Health Evaluation. Health Education. Delivery of Health Care. Public Health.

\section{INTRODUÇÃO}

O sistema de saúde brasileiro conta com uma legislação privilegiada que norteia a dinâmica dos serviços e das ações de saúde. A Lei Orgânica da Saúde (Lei 8.080/1990) coloca como princípios e diretrizes principais; a universalidade, a equidade, a integralidade, a descentralização e a participação social. Os compromissos são pactuados, envolvendo as três esferas de governo. ${ }^{1}$

O Ministério da Saúde, no sentido de priorização da gestão pública e da melhoria da atenção básica, está desenvolvendo ações em conjunto com estados e municípios, com base na indução, monitoramento, avaliação de processos e resultados na área da saúde, no sentido de garantir o acesso e a qualidade dos serviços à população. ${ }^{2}$ Entre elas, podem ser destacadas as ações da Política Nacional de Atenção Básica (PNAB) de 2011, que se caracteriza por um conjunto de ações de saúde no âmbito individual/coletivo, abrangendo a promoção e proteção de grupos vulneráveis, prevenção de agravos, diagnóstico, tratamento, manutenção da saúde, buscando promover atenção integrada. ${ }^{3}$

A portaria 1.654 do Ministério da Saúde, publicada em 19 de julho de 2011, instituiu o Programa Nacional de Melhoria do Acesso e da Qualidade da Atenção Básica (PMAQ-AB), que tem como seu principal objetivo induzir a ampliação do acesso e a melhoria da qualidade da atenção básica, com garantia de um padrão de qualidade comparável nacional, regional e localmente de maneira a permitir maior transparência e efetividade das ações governamentais direcionadas à Atenção Básica em Saúde (ABS). ${ }^{4}$ O programa está organizado em quatro fases 
que se complementam e constituem um ciclo continuo de melhoria do acesso e da qualidade, sendo a primeira a Adesão e Contratualização, a segunda o Desenvolvimento, a terceira a Avaliação Externa e a quarta a Recontratualização. A cada fase, parâmetros de qualidade são definidos. No primeiro ciclo do PMAQ-AB, os avaliadores e equipe se basearam em quarenta e sete indicadores de qualidade, parâmetros esses estabelecidos pelo Ministério da Saúde. Os primeiros resultados das avaliações onde os indicadores não foram atingidos pelas equipes, estas tiveram um prazo para se adequar as metas necessárias contemplando a exigência do Ministério da Saúde. ${ }^{4}$

Nessa linha de pensamento, busca-se responder as seguintes problemáticas: OPMAQ$\mathrm{AB}$ está sendo desenvolvido neste município? Os trabalhadores têm conhecimento e se utilizam desta metodologia para o planejamento e a avaliação das ações nas ESFs?

Justifica-se o presente trabalho pelos questionamentos que surgiram durante a vivência acadêmica na Rede Básica de Saúde deste município. Por diversos momentos de buscas nos serviços, as resposta não foram convincentes e motivaram o desenvolvimento de estudos e desta pesquisa. Estes fatos nos instigam à procura de informações no sentido de aprofundar o conhecimento.

Portanto, este estudo tem como objetivo investigar sobre a metodologia de aplicação do PMAQ-AB: sua avaliação pelos profissionais de saúde, seus resultados e seu impacto na organização e na assistência à saúde, sob a ótica dos trabalhadores da saúde.

\section{MÉTODOS}

O presente estudo faz parte da pesquisa "Aplicação do Programa Nacional de Melhoria do Acesso e da Qualidade da Atenção Básica (PMAQ-AB): olhar avaliativo dos profissionais de saúde" desenvolvido pelo Grupo de Estudos e Pesquisa em Saúde da Universidade de Santa Cruz do Sul (GEPS/UNISC).

A pesquisa está fundamentada em princípios éticos e de acordo com a Resolução 466/12, do Conselho Nacional de Saúde (CNS), regulamentadora de Pesquisas envolvendo Seres Humanos. ${ }^{5} \mathrm{O}$ projeto de pesquisa foi autorizado pela instituição parceira, $13^{\mathrm{a}}$ Coordenadoria Regional de Saúde/RS, submetido e aprovado pelo Comitê de Ética em Pesquisa da Universidade de Santa Cruz do Sul-UNISC, sob protocolo n ${ }^{1.171 .974 / 15 .}$

O local do estudo foi um município da $28^{\text {a }}$ Região de Saúde - RS. O mesmo conta com 17 ESF implantadas, dando uma cobertura de 48,88\% da população. Consta no Diário Oficial da União $\mathrm{N}^{\circ} 121$, do dia 29 de junho de 2015, a certificação de 11 Equipes de Atenção Básica, sendo que 8 destas, possuem Equipe de Saúde Bucal. ${ }^{6}$ 
A coleta de dados ocorreu em documentos na Secretaria de Saúde do município e através de entrevistas com 21 trabalhadores de saúde das unidades de ESFs. Anteriormente a coleta, foi solicitada autorização ao Secretário Municipal de Saúde para o desenvolvimento do estudo.

Na inclusão dos sujeitos, foram contemplados o gestor de saúde e um representante por categoria profissional em cada ESF que tenha participado em alguma fase do PMAQ-AB (enfermeiro, médico, cirurgião dentista, técnico de enfermagem, nutricionista) das unidades básicas de saúde deste município, envolvidas com o PMAQ-AB. Quando havia mais de um trabalhador da mesma categoria no local, a escolha foi aleatória. Como critério de exclusão, foi a não aceitação do sujeito em participar do estudo. Do total de 23 trabalhadores da saúde dos ESFs que constituíram a amostra, dois sujeitos, de ESFs distintas, não aceitaram participar. O Termo de Consentimento Livre e Esclarecido foi apresentado aos sujeitos para assinatura, após a apresentação do estudo.

As entrevistas ocorreram no período de abril a junho de 2016, conforme a disponibilidade, no horário de trabalho dos profissionais de saúde no ESF e foram agendadas previamente por contato telefônico. As mesmas continham questões norteadoras e foram gravadas e transcritas. Com o gestor não foi possível realizar a entrevista por questões de indisponibilidade, sendo que o mesmo indicou a enfermeira Coordenadora da Atenção Básica.

Com a transcrição das entrevistas, constituiu-se um texto contendo registro de todos os resultados, destacando-se as respostas significativas para os objetivos do estudo. No agrupamento das ideias, foram seguidas as orientações metodológicas da Análise de Conteúdo, organizando-as em temáticas. ${ }^{7}$ Os temas contemplados foram: PMAQ na visão dos trabalhadores; a metodologia de aplicação do PMAQ; conhecimento dos resultados da avaliação e mudanças nas unidades de saúde com a implantação do programa.

No corpo deste trabalho, são apresentadas algumas falas dos sujeitos pesquisados com a letra E, seguindo uma ordem cronológica de realização das entrevistas, como por exemplo, E1, E2, E3.

\section{RESULTADOS}

Foram 21 sujeitos pesquisados: dois médicos, cinco enfermeiros, sete técnicos de enfermagem, seis odontólogos e uma nutricionista. Destes, 14 tinham formação em ensino superior; quatro eram profissionais concursados e os demais contratados; com idade entre 27 e 59 anos, com tempo de atuação de 2 a 13 anos na rede de atenção básica; e carga horária de 40 horas semanais. 


\section{O PMAQ na visão dos trabalhadores da saúde}

Quando solicitados aos trabalhadores que falassem livremente sobre o Programa, os que possuíam conhecimento sobre o mesmo, interpretavam-no em dimensão avaliativa. [...] teve avalição da unidade, os avaliadores fizeram perguntas, avaliaram a unidade E9, E11.

Dois profissionais, técnicos em enfermagem, informaram que desconheciam esse programa conforme exposto nas falas a seguir:"não tenho o que falar porque não sei, assim que surgiu a história de PMAQ, foi um AUE.., disseram que seria um incentivo para ajudar a equipe, mas se apagou”'E10, “não temos PMAQ aqui, no outro posto de saúde tinha” E8.

Do total de entrevistados, três relataram ter participado de todas as fases do programa; quatro visualizam a qualidade do programa por ser uma política governamental com objetivo de humanizar a assistência e fortalecer a atenção primária.

Um trabalhador entende o PMAQ como a organização da unidade. Essa ideia é fortalecida na resposta de quatro trabalhadores que acrescentam que a melhor forma de fazêlo é lendo e estudando os manuais e apostilas do programa, como exposto nas falas a seguir: "eu vi toda a movimentação para organização da entrevista, correria uma semana antes em todas as unidades, precisava deixar tudo redondinho para os avaliadores olharem "E1; “ [...] gente tinha feito um estudo da apostila e quando a gente recebeu os avaliadores na unidade estava tudo em ordem, de acordo como a apostila mandava, todos os requisitos" E12.

A maioria dos trabalhadores enfatizou em suas colocações, a organização do trabalho e questões de infraestrutura, no que tange a compreensão sobre esse programa: " $a$ organização da unidade, de acordo com as regras do PMAQ serve para a organização da ESF"'E4.Na visão dos trabalhadores de saúde, a organização interna da unidade é o objeto principal da metodologia de implementação do PMAQ: "sim, a estrutura, é uma qualidade, tá melhorando a qualidade do atendimento, o usuário e os trabalhadores" E6-E7.

Alguns se referiram ao programa com lembranças de sua fase inicial, no entanto, não Oidentificam o mesmo em sua unidade de saúde: "eu percebo que o PMAQ teve uma ênfase bem maior no inicio, eu não tenho noção das fases do programa, eu me lembro de quando ele veio, quando foi instituído, ter tido muita mobilização, reunião [...] mas eu não tenho noção do que é dele aqui dentro do posto” E15.

\section{Metodologia de aplicação}

Ao questionar sobre a metodologia de aplicação do programa na unidade, três trabalhadores dizem não identificá-la, quatro se utilizam dos manuais do Ministério para 
atingir os indicadores, sete percebem a aplicação na organização do trabalho, dois apontam apenas o sistema informatizado da unidade e os demais não se pronunciaram: "Agente tenta seguir os requisitos que o PMAQ tem [...] não tenho dificuldade com os instrumentos” E7.

Quando questionados sobre como foram informados sobre o programa, relatam a realização de reuniões iniciais pela coordenação: “quando houve esse programa foi feito uma reunião com as equipes informando para que ele servia, foi passado para as equipes que seria interessante a gente entrar nesse programa e ai foi passado para cada equipe a cartilha” E21.

As informações sobre o programa aparecem nas falas de alguns trabalhadores como uma apresentação inicial, enfatizando a importância do mesmo. Porém, a continuidade do mesmo não aparece com a ênfase dada no início de sua implementação: “atualmente não tem sido colocado em prática, basicamente existem condições para isso mais não tem sido feito reuniões e traçado objetivos" E13; "Ultimamente não tem tido aplicação, a gente mal tem tempo de pensar em PMAQ, é muito atendimento, mas é valido porque direciona o trabalho" E9.

Observa-se o desconhecimento, dos entrevistados em relação à aplicação da metodologia, bem como seu uso no ambiente de trabalho, porém em algumas falas percebe-se que se utilizado de forma contínua, o programa contribui para a melhoria dos indicadores e seus resultados de avaliação.

\section{Conhecimento dos resultados da avaliação}

Quanto aos resultados das avaliações realizadas após a implantação do PMAQ, dez trabalhadores relatam não saberem dos mesmos; cinco tem conhecimento e outros cinco acrescentam que sabem dos resultados da primeira avaliação; e um não soube informar: "Não sei” E10-E11-E16; “Não tenho, não sei dizer” E4-E15; “não, nunca mais foi nos falado coisa referente ao PMAQ, se for feito realmente como prega o PMAQ, eu acho que é para melhorar o funcionamento, tanto para equipe, quanto para o usuário” E18.

Foi citada por alguns entrevistados a ocorrência de um evento de apresentação dos resultados pelas equipes das ESFs, momento em que os mesmos foram informados. Entretanto, a maioria não tem clareza quanto aos resultados obtidos em cada indicador avaliado: "o que foi nos passado é que atingimos todos os indicadores, a gente se preocuparia em melhorar nosso trabalho e ver o que a gente tem que dar prioridade" E1; "Assim desde que foi implantado, a gente consegui fazer, seguir algumas diretrizes, e principalmente a parte de organização, mas medir indicadores a gente não consegue” E20. 
Evidencia-se a falta de comunicação quanto aos resultados das avaliações, vários entrevistados não possuem informações sobre os mesmos, assim como, não têm visibilidade para a identificação da fase do programa que está acontecendo na unidade de trabalho. Os mesmos relatam a não devolução dos resultados por parte da gestão, gerando descontentamento. Também, não relacionam mudanças com a implantação do programa.

\section{Mudanças nas unidades de saúde com a implantação do programa}

Quando questionados sobre as mudanças que ocorreram com a implantação do programa nas unidades, sete dos entrevistados não observam mudanças na unidade, dois observam, mas não sabem identificar onde, oito apontam a melhoria na organização da unidade e quatro apontam melhorias na estrutura: “... não tem influenciado nas ações de saúde e nem na organização do trabalho"E2; "Não observo benefícios aos usuários e aos trabalhadores" E1-E11; "a unidade sempre continuou a mesma, os beneficiados deve ser a prefeitura, nós não” E10.

Grande parte dos trabalhadores relata não perceber mudanças nas suas unidades de trabalho após implantação do programa, alguns apontam como mudanças a melhoria na organização da unidade que trabalham, trazendo como beneficiados a equipe e usuários do serviço de saúde, como citam: “... a parte organizacional do posto, acho que todos os funcionários e usuários são beneficiados, porque vem uma verba do PMAQ né, para onde vai não sei te dizer"'E4- E2O.

No que se refere à estrutura, foi enfatizado nas falas as condições do ambiente da unidade, incluindo materiais e orientações de fluxo interno: "teve algumas mudanças, tipo sinalização, mas as estruturais de parede ficou no papel” E7; “se for ver em percentual, o quanto impactou eu não diria...mas eu acho que tem, a equipe como um todo acabou se educando...talvez esteja numa fase inicial, deveríamos ter avançado muito mais”'E20. É identificada a necessidade de intensificar a dinâmica de desenvolvimento do programa e a educação da equipe aparece como mudanças.

\section{DISCUSSÃO}

Os trabalhadores são considerados como agentes de mudanças no ambiente de trabalho em que estão inseridos, e a comunicação efetiva é primordial para que o processo de trabalho se desenvolva de forma continuada. O estudo aponta algumas fragilidades e carências no que tange a informação. Quando questionados os sujeitos pesquisados sobre o seu 
conhecimento em relação ao programa, trazem informações sobre a movimentação inicial do programa e o declínio com o passar dos anos.

A qualidade deve ser entendida como um processo em movimento, sendo que a atuação dos profissionais de saúde tem a capacidade de mudar o cenário de trabalho. $\mathrm{O}$ PMAQ é a principal estratégia indutora de mudanças nas condições e modos de funcionamento das unidades básicas de saúde, criando uma permanente e progressiva ampliação do acesso e da qualidade na atenção básica. A capacidade dos atores implicados em gerar condições para transformações, inclusive exigir adaptações em instrumentos que conduzam a evolução da política. ${ }^{8}$

O programa tem vários desafios a enfrentar, por ser uma política nova, com ênfase na melhoria do acesso e da qualidade, mantendo-se contínuo e pautado na sua metodologia de aplicação. ${ }^{4}$ Os entrevistados trazem como forma de justificar o não conhecimento da metodologia do programa, as rotinas de atendimento em suas unidades de trabalho, porém percebem o programa como algo positivo e facilitador.

Nessa linha de pensamento, o programa deve ser estudado em conjunto com outras políticas e programas com os quais se articula, uma vez que o papel do programa é construir um diagnóstico mais amplo que sua intervenção, ao ponto de orientar a intervenção de outras políticas, permitindo assim aos formuladores nacionais realizar correções de trajetórias. ${ }^{9}$

Durante a realização do estudo, ficou evidente falhas na comunicação entre a gestão e equipes de atenção básica, criando-se expectativas por parte dos trabalhadores, pois uma vez estando com o programa implantado em suas unidades de trabalho, e a partir da movimentação inicial, esperava-se retornos dos resultados e avaliação, para mudanças ou adequações na melhoria da assistência e do processo de trabalho.

O não reconhecimento do programa pelas equipes, e a falta de envolvimento, ocorre muitas vezes por não terem apoio suficiente e acesso as informações em tempo oportuno, a fim de conhecer o andamento das ações do programa, bem como o desconhecimento dos resultados das avaliações. A importância do retorno dessas informações, para os trabalhadores traria benefícios para melhoria da comunicação, participação na qualificação da assistência desses diversos atores, sendo que a gestão um agente disparador de mudançasl. ${ }^{9}$

Sobre os impactos ou mudanças nas unidades de saúde com a implantação do PMAQ, alguns entrevistados não percebem claramente mudanças, pois antes dessa política já existiam outras, com esse cunho de humanização e eles não conseguem identificar a origem de alguns benefícios. 
Apostando na mobilização dos atores locais o PMAQ é uma estratégia da PNAB, buscando gerar mudanças ao indicar as diretrizes, ações e resultados esperados. O programa é uma ferramenta que busca induzir a direção do movimento através de padrões de qualidade, apostando fortemente na mobilização, na dinâmica da atuação e negociação, sendo que a gestão é a grande impulsionadora de ações e mudanças permanentes. ${ }^{10}$

Constam ainda os desafios que o PMAQ-AB pretende enfrentar para a qualificação da atenção básica: precariedades da rede física; condições de trabalho inadequadas para os profissionais; qualificação dos processos de trabalho das equipes de $\mathrm{AB}$; pouca integração dos membros das equipes; elevada rotatividade dos profissionais; sobrecarga das equipes comprometendo a cobertura e qualidade das ações; pouca resolutividade nas práticas; financiamento reduzido e inadequado da $\mathrm{AB}{ }^{4}$

A partir da investigação sobre a metodologia de aplicação do PMAQ-AB e a avaliação dos profissionais de saúde na utilização da mesma e de seus resultados, pode-se concluir a existência de fragilidade e falta de conhecimento dos profissionais sobre o programa PMAQAB. Por se tratar de uma política nova e a comunicação não ser efetiva, grande parte dos entrevistados não consegue reconhecer a metodologia de aplicação do programa.

A falta de retorno dos resultados e avaliações gera descontentamentos nos trabalhadores da saúde, pois acreditam que deveria existir maior comunicação entre o gestor e as equipes, como o trabalho se desenvolve pautado em indicadores, facilitaria no delineamento de novos objetivos para melhorias.

O PMAQ-AB tornou-se uma das principais políticas do Ministério da Saúde, sendo que seu objetivo é de qualificar a Atenção Básica, garantindo um padrão de qualidade nas três esferas de poder. O impacto do PMAQ-AB na organização e na assistência à saúde, não é identificado pela maioria dos trabalhadores participantes deste estudo.

Recomendam-se estudos sistematizados, de forma continua e com maior detalhamento sobre os indicadores de avaliação deste programa e com uma participação ampliada dos trabalhadores e usuários da saúde.

\section{REFERÊNCIAS}

1. Brasil. Lei Federal $n^{0} 8.080 / 90$. Dispõe sobre as condições para a promoção, proteção e recuperação da Saúde, a organização e o funcionamento dos serviços correspondentes e dá outras providências [internet]. Brasília, 1990. Disponível em: http://www.planalto.gov.br/ccivil_03/Leis/L8080.htm. 
2. Ministério da Saúde (BR). Secretaria de Atenção à Saúde. Departamento de Atenção Básica. Autoavaliação para a Melhoria do Acesso e da Qualidade da Atenção Básica: AMAQ / Ministério da Saúde. Secretaria de Atenção à Saúde. Departamento de Atenção Básica. - Brasília (DF). Ministério da Saúde, 2012. 134 p- (Série B. Textos básicos de saúde).

3. Ministério da Saúde (BR). Portaria 2.488, de 21 de outubro de 2011. Aprova a Política Nacional de Atenção Básica, estabelecendo a revisão de diretrizes e normas para a organização da Atenção Básica, para a Estratégia Saúde da Família (ESF) e o Programa de Agentes Comunitários de Saúde (PACS).

4. Ministério da Saúde (BR). Portaria 1.654, de julho de 2011. Institui, no âmbito do Sistema Único de Saúde, o Programa Nacional de Melhoria do Acesso e da Qualidade da Atenção Básica (PMAQ-AB) e o Incentivo Financeiro do PMAQ-AB, denominado Componente de Qualidade do Piso de Atenção Básica Variável - PAB Variável.

5. Conselho Nacional de Saúde. Resolução no 466, de 12 de dezembro de 2012. Aprova diretrizes e normas regulamentadoras de pesquisa envolvendo seres humanos. [Internet]. 2012. Disponível: http://conselho.saude.gov.br/resolucoes/2012/Reso466.pdf.

6. Departamento de Atenção Básica. Unidade Geográfica. [Internet]Município de Santa Cruz do Sul/RS Jan/Nov. 2015. Disponível em: http://dab.saude.gov.br/dab/historico_cobertura_sf/historico_cobertura_sf_relatorio.php.

7. Minayo MCS. O desafio do conhecimento: pesquisa qualitativa em saúde. 14. Ed. São Paulo: HUCITEC, 2014. 407 p.

Pinto HA, Souza A, Florêncio AR. O programa nacional de melhoria do acesso e da qualidade da atenção básica: reflexões sobre o desenho e processo de implantação. Rev Eletr Com, Informação Inov Saúde 2012; 6 (2). doi:http://dx.doi.org/10.3395/reciis.v6i2.492 páginas.

8. Pinto HA, Ferla AA. Formulação e implementação de políticas como pedagogias para a gestão: um ensaio a partir de três casos do sistema único de saúde. Saúde em redes 2015; 1 (1): 81-94.

9. Pinto HA, Souza AN, Ferla AA. O programa nacional de melhoria do acesso e da qualidade da atenção básica: várias faces de uma política inovadora. Saúde Debate 2014; 38 (n.especial): 358-372. 